\title{
Prebiotic Chemistry of the Hadean Earth Under the Young Active Sun
}

\author{
VLADIMIR S. AIRAPETIAN ${ }^{1}$, MENG JIN ${ }^{2}$, JUNXIANG \\ $\mathrm{HU}^{3}$, GANG LI ${ }^{3}$, BENJAMIN HAYWORTH ${ }^{4}$, KENSEI \\ KOBAYASHI $^{5}$, GUILLAUME GRONOFF ${ }^{6}$ AND JAMES \\ KASTING $^{7}$
}

${ }^{1}$ NASA Goddard Space Flight Center

${ }^{2}$ SETI

${ }^{3}$ University of Alabama, Huntsville, AL

${ }^{4}$ Penn State University

${ }^{5}$ Yokohama National University

${ }^{6} \mathrm{NASA}$ LaRC and SSAI

${ }^{7}$ Penn State

Presenting Author: vladimir.airapetian@nasa.gov

Is life unique to Earth or a common phenomenon in the Solar System and the Universe? This fundamental question is among the greatest puzzles of modern science. Earth's evident long-term habitability makes it a key data point for understanding the formation of habitable worlds in the Universe. Earth could be a special outlier, or it could be a typical rocky planet. Understanding the conditions that allowed for the emergence of life on early Earth, and whether other inner planets in our Solar System possibly also supported habitable conditions early in their histories but failed to sustain them due to their unique evolutionary trajectories, is a promising way to address these questions. For this, we need to know how the basic requirements for life as we know it such as liquid water, organic compounds and persistent external energy fluxes promoted the emergence and complexification of biological systems on early Earth and how they were impacted by planetary and solar properties. The early Solar System was a chaotic place, likely subject to frequent large impacts as well as the violently changing energetic ionizing radiation flux from the young Sun. In this talk, I will describe our observationally constrained state-of-the-art theoretical models of the corona, the wind and transient events from the young Sun at the time when life arose on Earth and discuss the impact of solar X-ray, Extreme UV and particle fluxes associated with superflares on our planet. Specifically, I will use these constrained energy fluxes to describe our recent atmospheric chemistry models impacted by energetic particles from the young Sun and formation and precipitation of biologically relevant molecules including hydrogen cyanide and formaldehyde to the Earth's surface. I will then highlight our recent results of laboratory experiments of irradiation of mildly reduced gas mixture irradiated by high energy protons with the fluxes consistent with our theoretical models and their implications to the formation of complex molecules on the Hadean Earth. 